\title{
SOLUTIONS OF SOME $L(2,1)$-COLORING RELATED OPEN PROBLEMS
}

\author{
Nibedita Mandal and Pratima Panigrahi \\ Department of Mathematics \\ Indian Institute of Technology Kharagpur \\ India \\ e-mail: nibedita.mandal.iitkgp@gmail.com \\ pratima@maths.iitkgp.ernet.in
}

\begin{abstract}
An $L(2,1)$-coloring (or labeling) of a graph $G$ is a vertex coloring $f$ : $V(G) \rightarrow Z^{+} \cup\{0\}$ such that $|f(u)-f(v)| \geq 2$ for all edges $u v$ of $G$, and $|f(u)-f(v)| \geq 1$ if $d(u, v)=2$, where $d(u, v)$ is the distance between vertices $u$ and $v$ in $G$. The span of an $L(2,1)$-coloring is the maximum color (or label) assigned by it. The span of a graph $G$ is the smallest integer $\lambda$ such that there exists an $L(2,1)$-coloring of $G$ with span $\lambda$. An $L(2,1)$-coloring of a graph with span equal to the span of the graph is called a span coloring. For an $L(2,1)$-coloring $f$ of a graph $G$ with span $k$, an integer $h$ is a hole in $f$ if $h \in(0, k)$ and there is no vertex $v$ in $G$ such that $f(v)=h$. A no-hole coloring is an $L(2,1)$-coloring with no hole in it. An $L(2,1)$-coloring is irreducible if color of none of the vertices in the graph can be decreased to yield another $L(2,1)$-coloring of the same graph. A graph $G$ is inh-colorable if there exists an irreducible no-hole coloring of $G$. Most of the results obtained in this paper are answers to some problems asked by Laskar et al. [5]. These problems are mainly about relationship between the span and maximum no-hole span of a graph, lower inh-span and upper inh-span of a graph, and the maximum number of holes and minimum number of holes in a span coloring of a graph. We also give some sufficient conditions for a tree and an unicyclic graph to have inh-span $\Delta+1$.
\end{abstract}

Keywords: $L(2,1)$-coloring, span of a graph, no-hole coloring, irreducible coloring, unicyclic graph.

2010 Mathematics Subject Classification: 05C15. 
[1] P.C. Fishburn and F.S. Roberts, No-hole L(2,1)-colorings, Discrete Appl. Math. 130 (2003) 513-519. doi:10.1016/S0166-218X(03)00329-9

[2] J.P. Georges, D.W. Mauro and M.A. Whittlesey, Relating path coverings to vertex labellings with a condition at distance two, Discrete Math. 135 (1994) 103-111. doi:10.1016/0012-365X(93)E0098-O

[3] J.R. Griggs and R.K. Yeh, Labelling graphs with a condition at distance 2, SIAM J. Discrete Math. 5 (1992) 586-595. doi: $10.1137 / 0405048$

[4] R. Laskar and G. Eyabi, Holes in L(2,1)-coloring on certain classes of graphs, AKCE Int. J. Graphs Comb. 6 (2009) 329-339.

[5] R.C. Laskar, J. Jacob and J. Lyle, Variations of graph coloring, domination and combinations of both: a brief survey, Advances in Discrete Mathematics and Applications, Ramanujan Mathematical Society Lecture Notes Series 13 (2010) 133-152.

[6] R.C. Laskar, G.L. Matthews, B. Novick and J. Villalpando, On irreducible no-hole $L(2,1)$-coloring of trees, Networks 53 (2009) 206-211. doi: $10.1002 /$ net.20286

[7] R.C. Laskar and J.J. Villalpando, Irreducibility of L(2,1)-coloring and inh-colorablity of unicyclic and hex graphs, Util. Math. 69 (2006) 65-83.

[8] W.F. Wang, The L(2,1)-labelling of trees, Discrete Appl. Math. 154 (2006) 598-603. doi:10.1016/j.dam.2005.09.007

[9] D.B. West, Introduction to Graph Theory (New Delhi, Prentice-Hall, 2003).

[10] M.Q. Zhai, C.H. Lu and J.L. Shu, A note on L(2,1)-labelling of trees, Acta Math. Appl. Sin. Engl. Ser. 28 (2012) 395-400.

doi:10.1007/s10255-012-0151-9

Received 21 January 2015

Revised 15 June 2015

Accepted 15 June 2015 